\title{
Trayectorias estudiantiles en contexto de pobreza socioeconómica: una aproximación desde la región Bío Bío, Chile
}

\begin{abstract}
Students Trajectories in the contexts of socioeconomic poverty: an approach from Bio Bío Región, Chile
\end{abstract}

\section{Volumen 21, Número 1 \\ Enero - Abril \\ pp. 1-25}

Marcela Irene Suckel Gajardo

María Teresa Chiang Salgado

\section{Citar este documento según modelo APA}

Suckel Gajardo, Marcela Irene. y Chiang Salgado, María Teresa. (2021). Trayectorias estudiantiles en contexto de pobreza socioeconómica: una aproximación desde la región Bío Bío, Chile. Revista Actualidades Investigativas en Educación, 21(1), 1-25. Doi. 10.15517/aie.v21i1.42608 


\section{Trayectorias estudiantiles en contexto de pobreza socioeconómica: una aproximación desde la región Bío Bío, Chile \\ Students Trajectories in the contexts of socioeconomic poverty: an approach from Bio Bío Región, Chile}

\section{Marcela Irene Suckel Gajardo ${ }^{1}$ María Teresa Chiang Salgado ${ }^{2}$}

Resumen: Este artículo se realizó para conocer las trayectorias de vida de estudiantes de liceos en contextos de pobreza socioeconómica. Se trata de un abordaje cualitativo y biográfico, realizado entre los años 2015 y 2016 , en nueve liceos de la provincia de Bío Bío, Chile. Desde un enfoque biográfico fue posible acceder a las trayectorias educativas de 180 jóvenes de secundaria a través de los relatos de vida de los y las participantes. Los hallazgos principales muestran tres tipos de trayectoria: definidas, difusas e indefinidas según establecimiento de metas $y$ proyecciones de vida identificadas por el estudiantado. Estas trayectorias se diferencian de acuerdo con las expectativas de logro personal, laboral y/o académico, creencias y sintonía afectiva ligada a las creencias. Los principales hallazgos revelan trayectorias diversificadas según el procesamiento reflexivo en torno al proceso de construcción de proyectos. Característico de esta generación sería el sentimiento de pragmatismo, ven de manera práctica y vivencial si sus expectativas optimistas tienen correlato con sus experiencias de vida. Se produce, entonces, un desajuste entre esas altas expectativas, las que ven difícil de concretar, por los desiguales accesos a las oportunidades sociales, por lo que la forma de resolver la complejidad en sus trayectorias daría cuenta de reflexiones diversas que colisionan con experiencias de vida ligadas a desventajas estructurales.

Palabras clave: estudiante de secundaria, pobreza, exclusión, trayectoria educativa, enfoque biográfico.

Abstract: This article was made to find out the life trajectories of high school students in contexts of socioeconomic poverty. It is a qualitative and biographical approach, carried out between 2015 and 2016, in nine high school in the province of Bío Bío, Chile. From a biographical approach, it was possible to access the educational trajectories of 180 secondary school youth through the life stories of the participants. The main findings show three types of trajectory: defined, diffuse, and undefined according to the establishment of goals and life projections identified by the student body. These trajectories are differentiated according to expectations of personal, work and / or academic achievement, beliefs and affective attunement linked to beliefs. The main findings reveal diversified trajectories according to reflective processing around the process of construction of projects. Characteristic of this generation would be the feeling of pragmatism; they see in a practical and experiential way if their optimistic expectations are correlated with their life experiences. There is, then, a mismatch between these high expectations, which they find difficult to specify, due to the unequal access to social opportunities, so that the way to resolve the complexity in their trajectories, would account for diverse reflections that collide with life experiences linked to structural handicaps.

Key words: high school student, poverty, exclusion, educational trajectory, biographical approach.

\footnotetext{
${ }_{1}$ Profesora Universidad de Concepción, Departamento de Currículum e Instrucción, Bío Bío, Chile. Dirección electrónica: marcelasuckel@udec.cl, ORCID https://orcid.org/000$\underline{00018576-3346}$

${ }^{2}$ Profesora Universidad de Concepción, Departamento de Fisiopatología, Bío Bío, Chile. Dirección electrónica: mchiang@udec.cl ORCID https://orcid.org/000-0003 3499-6291
}

Artículo recibido: 30 de junio, 2020

Enviado a corrección: 11 de setiembre, 2020

Aprobado: 9 de diciembre, 2020 


\section{Introducción}

Este artículo aborda las trayectorias de estudiantes de secundaria provenientes de sectores de pobreza socioeconómica a partir de una aproximación al devenir de sus proyectos en un contexto de segregación escolar. En Chile, durante las últimas tres décadas, el sistema escolar ha experimentado transformaciones a través de la introducción de mecanismos de mercado, como el financiamiento mediante voucher y políticas públicas que han promovido el aumento de años de escolarización, transformaciones curriculares y mejoramiento en la formación inicial docente (Bellei, 2015).

Un hito relevante se produjo el año 2003 cuando se estableció la escolaridad obligatoria la que se extendió de 8 a 12 años (Ley 19.876, 2003) y que rige para el estudiantado hasta los 21 años. Esta ley muestra el compromiso del Estado para asegurar la oferta educativa en todo el territorio, con el propósito de que todo el estudiantado finalice su educación secundaria.

Las políticas públicas, sin embargo, no han transformado dos características del sistema escolar: la segregación y la desigualdad escolar. De acuerdo con la Organización para la Cooperación y el Desarrollo Económicos (OCDE) "el sistema educacional está conscientemente estructurado por clases" (OCDE, 2004, p. 277), caracterizado por un nivel elevado de segregación escolar (Valenzuela et al., 2010). La segregación escolar es producto de aspectos estructurales de la sociedad y de características de la organización y funcionamiento del sistema educacional (Valenzuela, et al. 2010), como son el financiamiento tipo voucher, copago de las familias, entre otras, que presentan una fuerte orientación de mercado (Bellei, 2013). Estos aspectos se encuentran estrechamente relacionados, pues "la segregación socioeconómica y académica de la población escolar constituyen factores relevantes de la (in)equidad educativa, en tanto afectan diferencialmente las oportunidades y logros de aprendizaje de los alumnos" (Bellei, 2013, p. 339).

Espínola, Claro y Walker (2009) destacan la segmentación según dependencia administrativa de los establecimientos escolares (particular, subvencionado y municipal) relacionada al nivel socioeconómico de las familias, proceso que contribuiría a perfilar trayectorias educativas altamente predictibles según el nivel de ingresos de cada estudiante. A grupos escolares de altos ingresos se les abren oportunidades mientras que a grupos escolares de bajos ingresos se les van cerrando de manera acumulativa, dependiendo, de los eventos críticos tempranos, tales como repitencias, deserción entre otros (Espínola, Claro y Walker, 2009). La educación de jóvenes pobres, provenientes de establecimientos municipalizados, tiende a reproducir los circuitos de la pobreza en una espiral que aleja a 
algunos sectores de la educación secundaria, por lo que el liceo en vez de atenuar las desigualdades las profundiza (García-Huidobro, 1999).

En contraste, el acceso a la educación superior del estudiantado ha experimentado un aumento constante (Brunner, 2009; Espinoza, 2015; Zapata, Tejeda y Rojas, 2011), para lo cual, la mayoría de las universidades exige rendir la Prueba de Selección Universitaria (P.S.U.); uno de los requisitos para postular a la educación universitaria lo constituye obtener un puntaje mínimo de 450 puntos. La PSU, como prueba de selección, evalúa habilidades cognitivas generales y tiene por finalidad ordenar a los egresados de la educación secundaria según las habilidades medidas, por lo que analizar sus resultados y determinantes representaría un modo de evaluar la equidad de los resultados del sistema educacional chileno en cuanto a su capacidad para asegurar el aprendizaje del estudiantado con independencia de su origen social y su capacidad para asegurar igualdad de oportunidades en el acceso a educación superior (Contreras, Corbalán y Redondo, 2007). Estos resultados revelan factores socioeconómicos y familiares asociados al acceso desigual de jóvenes a la educación superior (Contreras et al., 2007) y una "brecha significativa y creciente" en los puntajes de la PSU (Muñoz y Redondo, 2013, p. 117).

La desigualdad en el acceso se expresa también en las diferencias por ingresos de jóvenes que acceden a la educación superior. El estudiantado perteneciente al decil I de ingresos presentan una tasa neta de 28.4, mientras que los del decil $X$ alcanzan una tasa neta de 61.1, lo cual duplica a los primeros en referencia al porcentaje de nuevos entrantes al nivel de educación superior (Gobierno de Chile-CASEN 2015). La preferencia por tipo de institución también se diferencia por ingresos: jóvenes de los primeros dos deciles de ingreso son la principal matrícula de centros de formación técnica e institutos profesionales que ofrecen carreras más acotadas en tiempo y coste económico que las universidades según la encuesta de Caracterización Socioeconómica Nacional (Gobierno de Chile-CASEN 2015).

\section{Referente teórico}

La investigación se orientó a partir de los conceptos de trayectorias y proyectos. Dubar (1998) plantea que el análisis de las trayectorias se articula en torno a dos aspectos de los procesos biográficos. La trayectoria "objetiva", vinculada a las posiciones sociales ocupadas durante la vida, medidas por medio de categorías estadísticas; y la trayectoria "subjetiva" expresada en las "historias personales" cuyo relato remite a las visiones del mundo y de sí mismo (Dubar 1998, p. 73). Respecto de la trayectoria "objetiva”, Doray (2012) sostiene que 
el concepto de trayectoria está asociado a las teorías de la reproducción. Las trayectorias recorridas por los individuos son trayectorias asociadas a la clase, aunque la pertenencia social desemboque en un conjunto de trayectorias posibles. Las trayectorias sociales tienen un punto de inicio definido por el volumen y la estructura de capitales con que cuenta el individuo al nacer y es la familia el factor determinante y del que depende el patrimonio de capital heredado, condiciones materiales de existencia, lugar de residencia (Dávila, Ghiardo y Medrano, 2005). Así el origen social es el elemento principal en la determinación de las trayectorias individuales (p. 58).

De acuerdo con Rubilar, Muñiz-Terra y Domínguez (2019), la subjetividad es posible comprenderla como apropiaciones e imaginarios que el estudiantado hace de sus configuraciones identitarias en interacción con otros y su entorno, observables en las trayectorias que configuran en en interacción con las estructuras y discursos sociales.

En el caso de las trayectorias escolares, Terigi (2009) ha diferenciado las trayectorias teóricas y trayectorias reales. Siguiendo a la autora, las trayectorias teóricas expresan una progresión lineal de los itinerarios estudiantiles en el sistema escolar, prevista por una estructura asociada a tres elementos clave: la organización a través de niveles, la gradualidad del currículum y la anualización de los grados de instrucción. Por su parte, las trayectorias reales se identifican una vez analizadas las trayectorias de las personas donde se reconoce la heterogeneidad y contingencia con las que cursa la escolarización del estudiantado, marcada por fenómenos como la repitencia y el abandono escolar (Terigi, 2009). En este sentido, para conocer las trayectorias educativas se requiere analizar aspectos como las condicionantes del grupo social de origen, el tipo de escuela en que se cursa, el valor de los certificados que se ofrecen y el valor simbólico de obtenerlos, entre otros (Dávila y Ghiardo, 2005). Dado lo anterior, los individuos no se desplazan al azar en el espacio social, sino más bien en relación con un determinado volumen de capital (Bourdieu, 1984).

Canales, Opazo y Camps (2016) plantean la existencia de un "proyecto generacional" en el estudiantado, que consiste en ser más, transformarse en alguien, aspiración compartida con sus familiares, y que otorga sentido a seguir estudiando. La experiencia de los padres y las madres, relato familiar que cruza las generaciones, marca profundamente a jóvenes que, como primera generación con acceso a poder «ser alguien», son depositarios del encargo familiar de «reparar» en sus vidas las carencias y sufrimientos de sus progenitores (Canales, et al., 2016, p. 99). Por lo que en consideración de que el habitus reproduce las condiciones sociales de las que es producto, también es posible mencionar que las prácticas disruptivas y 
transformadoras son posibles cuando el desarrollo de nuevas condiciones históricas genera la oportunidad de reorganizar las disposiciones adquiridas (Gluz, 2006).

Desde esta perspectiva, el actor construye una racionalidad en función de sus objetivos, recursos y posiciones, y define una identidad por lo que es, lo que posee y los intereses que se tengan (Dubet y Martuccelli 1998).

Dar cuenta de la racionalidad construida por estudiantes es el propósito del presente estudio, para lo cual se exploran sus trayectorias vocacionales a partir de la elaboración de expectativas de corto, mediano y largo plazo, y el proceso de vinculación afectiva de las personas en la proyección que realizan de sus vidas, en las dimensiones personal y social.

El entorno familiar es un elemento importante en la estabilidad de las trayectorias de jóvenes tendientes o no al ingreso a la universidad (McCulloch, 2017). Baker et al. (2014) encontraron que, a pesar de las altas aspiraciones, existen diferencias por ingresos y otros factores estructurales; así, el estudiantado de entornos menos favorecidos tiene aspiraciones más bajas que sus pares más favorecidos. Los hallazgos indican que las aspiraciones estudiantiles dan cuenta de las oportunidades disponibles, es decir, están vinculadas a la probabilidad de alcanzarlas. Anders (2017) señala que jóvenes de todos los estatus socioeconómicos comienzan su adolescencia con altas expectativas de postular a la universidad. No obstante, aquellos de entornos menos favorecidos son más propensos a revisar sus expectativas a la baja y, de igual modo, poseen menos probabilidades de aumentar sus expectativas a medida que finalizan la educación secundaria. De forma complementaria, Hopenhayn y Ottone (2000), plantean que la educación secundaria, tanto en su modalidad científico-humanista como en la técnico-profesional vive una situación de crisis por la masificación de la educación secundaria que la hace perder su carácter de puente hacia la élite y la rentabilidad ocupacional fuertemente cuestionada de la educación técnica profesional.

Junto al explosivo aumento de la matrícula en educación superior, acaecida durante los últimos 25 años, las expectativas estudiantiles de estudiar en la universidad se han ampliado de manera consistente. Respecto a la masificación de la educación media y su influencia en los proyectos de futuro del estudiantado, escenarios de competencia educativa y social propiciarían condiciones percibidas desde la obligatoriedad en las personas a generar estrategias y a utilizar racionalmente el sistema educativo, con lo cual, la idea de proseguir estudios superiores se expresa como una obligación si se aspira a posicionarse en el mercado laboral (Dubet y Martuccelli 1998; Cit. en Castillo y Cabezas 2010, p. 50). 
En la Encuesta a Actores del Sistema Educativo aplicada a 1888 jóvenes estudiantes de cuarto año de educación secundaria de 69 establecimientos educativos de la región Metropolitana, Chile (Sevilla, 2012; cit. en Sepúlveda y Valdebenito 2014a, p. 1), un 43\% del estudiantado manifestó que su principal expectativa era estudiar en la universidad. Sin embargo, existen diferencias de acuerdo con el tipo de establecimiento: en establecimientos municipales, la cifra alcanza el 29\% del estudiantado; en particulares subvencionados, un $41 \%$; y en particulares pagados, un $68 \%$. Asimismo, se ha producido un aumento de las aspiraciones para obtener un título universitario en estudiantes de la modalidad técnicoprofesional, tradicionalmente orientada al mundo del trabajo. Debido a las dificultades que visualizan, tanto a nivel económico como académico, el estudiantado proyecta combinación de estudios superiores y trabajo, lo que revela su capacidad de elaborar un proyecto educativolaboral de futuro (Sepúlveda y Valdebenito, 2014b) y de realizar ajustes entre las expectativas y las posibilidades de alcanzarlas, que se vinculan con las condiciones económicas y las futuras exigencias académicas que visualizan enfrentarán en el mediano plazo.

Este grupo de estudiantes manifiesta opciones menos exigentes en términos académicos. Las universidades no selectivas constituyen una alternativa realista en función de las dificultades para obtener un alto puntaje PSU. De tal forma, la elección de una institución de educación superior y el capital cultural revelarían una reproducción de la segmentación de los establecimientos escolares donde estudiaron. En consideración de elementos de la reproducción social, se evidencia una superposición de desigualdades que se entrecruzan, por lo que a las desigualdades iniciales se suma la ausencia de referentes para poder construir un futuro en escenarios de mayor incertidumbre y transformaciones del mercado del trabajo, así como la falta de un proyecto crítico que observe dichas desigualdades (Rubilar et al., 2019).

A nivel de políticas públicas, dos programas recientemente implementados tienen relación con el fenómeno analizado: un primer programa asociado a la gratuidad de los estudios superiores y el programa PACE. Tanto el programa asociado a la gratuidad como el programa PACE, a través del apoyo económico y académico, buscan generar impactos favorables en el estudiantado de clase baja, en un intento de entregar igualdad de oportunidades. Al respecto, el presente estudio-pretende aportar distinciones y diferenciaciones de identidad emanadas desde personas intervenidas con las políticas públicas recientemente mencionadas. Al atender al sujeto social y las diversas realidades socioculturales en que se insertan se posibilitaría la incorporación de aspectos identitarios y de construcción social del conocimiento del estudiantado de educación secundaria. 
Por lo anteriormente señalado, el objetivo de este estudio fue analizar las trayectorias de estudiantes de secundaria de liceos en contextos de pobreza socioeconómica de las provincias de Concepción y Bío Bío, Chile.

\section{Metodología}

El artículo presenta una investigación acerca de las trayectorias de estudiantes procedentes de Chile, de las provincias de Concepción y Bío Bío. Las preguntas que lo orientaron fueron: ¿Cuáles son las trayectorias de estudiantes de secundaria de establecimientos educacionales insertos en contexto de pobreza socioeconómica? ¿Cuáles son las definiciones y diferenciaciones que establece el estudiantado en el proceso de elaboración de expectativas respecto de su futuro? Desde una aproximación cualitativa y biográfica fue posible acceder a los trayectos educativos de jóvenes de secundaria a través de los relatos de vida de las personas participantes.

El presente estudio se realizó en nueve liceos, insertos en sectores de pobreza socioeconómica, cuya ubicación geográfica correspondió a las provincias de Concepción y Bío Bío, Chile. Todos estos establecimientos participaron como beneficiarios de un programa de intervención estatal del Ministerio de Educación [Mineduc] (2015) implementado por la Universidad de Concepción, Chile desde el año 2015 a la fecha, cuyo propósito es el acompañamiento, acceso efectivo y restitución del derecho a la educación superior para el estudiantado que finaliza el ciclo de escolarización secundaria obligatoria.

\subsection{Enfoque}

Esta investigación es de tipo cualitativo de alcance exploratorio y se realizó mediante el enfoque biográfico, específicamente, la utilización del relato de vida, lo que permitió aproximarse a las construcciones identitarias de las personas. Los relatos configuran definiciones y diferenciaciones con los demás, lo que permite dar sentido a las acciones y a los eventos vividos. Desde un punto de vista ético, el relato de vida implica la toma de posición respecto de lo contado (Cornejo, Mendoza y Rojas, 2008). A nivel epistemológico, se establece una relación sujeto-sujeto en la que "el narrador no es el único que habla, piensa y se transforma" (Bertaux, 1993; Cit. en Cornejo et al. 2008, p. 31), sino que también el oyente o narrador realiza un aporte desde su posición.

La elección del enfoque biográfico se fundamentó en la coherencia que se establece entre este y la problemática en estudio al permitir conciliar lo social y lo psicológico, la 
subjetividad de la narración como fuente de conocimiento, expresión y construcción de la identidad (Bertaux, 1999; Cornejo, 2006; Cornejo et al., 2008).

\subsection{Participantes}

La población del estudio la constituyeron 180 escolares que cursaban el Tercer año de Enseñanza Media y que eran beneficiarios del programa PACE de las provincias de Concepción y Bio Bío, Chile, y en sectores de pobreza socioeconómica, condición que aumenta las dificultades para mantener altas expectativas de proseguir estudios superiores. El estudiantado participante del estudio fue el matriculado en 22 establecimientos educacionales de la región del Bío Bío, Octava Región, Chile, seleccionado por el Gobierno de Chile por su condición de establecimiento vulnerable, en consideración del nivel socioeconómico del estudiantado y su familia. El Programa de Acompañamiento Efectivo a la Educación Superior (PACE), se orienta a brindar acompañamiento, acceso efectivo y restituir el derecho a la educación superior a estudiantes que se encuentran en una etapa cercana a la finalización del ciclo de escolarización obligatoria. Con el fin de desarrollar esta iniciativa, se asignaron recursos a la Universidad de Concepción para apoyar al alumnado de tercero y cuarto año de enseñanza media. Este programa consideraba a las provincias de Concepción y Bío Bío con un número total de 570 estudiantes beneficiarios.

En este estudio se seleccionaron nueve establecimientos educacionales pertenecientes a la provincia de Bío Bío. Octava Región, Chile. Como criterio de selección se consideró la condición de vulnerabilidad de estos colegios, lo cual permitiría explorar las proyecciones futuras que tenía este estudiantado que vivía en condiciones de pobreza socioeconómica. La Tabla 1 detalla información de los establecimientos educacionales y sus participantes, en específico, el número de estudiantes según la modalidad de los liceos participantes (CientíficoHumanista, Técnico Profesional y Polivalente) y el Índice de Vulnerabilidad Escolar, I.V.E. (Junta Nacional de Auxilio Escolar y Becas [JUNAEB], 2017). 
Tabla 1

Número y caracterización de los liceos participantes según modalidad e Índice de Vulnerabilidad Escolar, Concepción, Bío Bío, Chile, año 2015

\begin{tabular}{|l|c|c|c|c|c|c|}
\hline \multirow{2}{*}{ Provincia } & \multicolumn{3}{|c|}{ Modalidad de los liceos participantes } & $\begin{array}{c}\text { Liceos } \\
\text { participantes }\end{array}$ & $\begin{array}{c}\text { Estudiantes } \\
\text { participantes }\end{array}$ & IVE-. SINAE 3 \\
\cline { 2 - 7 } & Humanifico- & $\begin{array}{c}\text { Técnico- } \\
\text { profesional }\end{array}$ & Polivalente & & & \\
\hline $\begin{array}{l}\text { Concepció } \\
\text { n }\end{array}$ & 1 & 1 & 3 & 5 & 100 & $88.7 \%$ \\
\hline Bio-Bio & 1 & 3 & 0 & 4 & 80 & $87.2 \%$ \\
\hline Total & $\mathbf{2}$ & $\mathbf{4}$ & $\mathbf{3}$ & $\mathbf{9}$ & $\mathbf{1 8 0}$ & $\mathbf{8 7 . 9 \%}$ \\
\hline
\end{tabular}

Fuente: Elaboración propia, con información del programa de Acceso y Acompañamiento Efectivo a la Educación Superior, PACE, 2015.

\subsection{Técnicas de recolección}

Para la elaboración de los relatos de vida, se utilizó la modalidad de encuentros con estudiantes de aproximadamente una hora de duración. Estos encuentros se realizaron durante el período de intervención del programa estatal P.A.C.E. que comprendió los años 2015 y 2016 , en sus respectivos liceos, previo a la firma de un consentimiento informado. Los intervalos entre el primer y segundo encuentro fluctuaron entre uno y tres meses, y la transcripción fue realizada sin editar, en el intento de ser lo más fiel posible con el relato escrito.

El foco específico en estudiantes de enseñanza media se asocia con la distribución normal de los talentos académicos, técnicos, pedagógicos, artísticos y científicos estudiantiles en todos los sectores de la sociedad independiente de su etnia y/u origen socioeconómico.

\subsection{Procesamiento de análisis}

Para la elaboración de los relatos de vida, se utilizó la modalidad de encuentros con el estudiantado, estos encuentros tuvieron una hora de duración aproximadamente. Los encuentros se realizaron entre el período lectivo y de intervención del programa estatal que

\footnotetext{
${ }^{3}$ IVE corresponde al índice de vulnerabilidad escolar en el marco del Sistema Nacional de Asignación con Equidad. SINAE está "definido como un conjunto de criterios que permite identificar distintos grupos dentro de la población de estudiantes de educación básica y media de establecimientos municipales o particulares subvencionados del país, de acuerdo al nivel de vulnerabilidad que presentan" (JUNAEB, 2017).

${ }^{4}$ Establecimiento escolar que ofrece educación científico-humanista y técnico-profesional.
} 
comprendió los años 2015 y 2016, en sus respectivos liceos, en salas facilitadas por cada establecimiento y en un horario acordado, previo a la firma de un consentimiento informado.

En un primer encuentro con los participantes, fueron invitados e invitadas a conversar acerca de sus vivencias y proyecciones asociadas a su paso por el liceo, sin restringir experiencia escolar alguna.

Durante el segundo encuentro, se instó a los participantes a plasmar las vivencias y proyecciones de vida por escrito, mediante un relato de vida de sus experiencias asociadas al liceo y cómo las proyectaban en el tiempo, tanto en términos educativos como laborales. Dada la proximidad temporal a la finalización de la enseñanza media, se invitó al estudiantado a reflexionar en torno a su trayectoria vocacional desde una mirada retrospectiva del proceso educativo y a proyectarse en las dimensiones personal y social. Para invitar a la reflexión y posibilitar la elaboración de una producción escrita se utilizó la consigna: "Cuéntanos cómo te ves en cinco años...".

Desde el enfoque biográfico, diversos autores plantean la inexistencia de un método único para el análisis de los datos (Lainé, 1998, Legrand, 1993 en Cornejo et al., 2008, p. 37), sino que el análisis se define de acuerdo con los objetivos de la investigación y del fenómeno estudiado. Para este estudio, resulta fundamental acceder a la comprensión del estudiantado en relación con sus vivencias y proyecciones de vida. Este aspecto constituye el punto de partida para categorizar y analizar los relatos en cuanto a las construcciones identitarias de las personas participantes del estudio. Mediante el desarrollo de una matriz de análisis de contenido de los relatos, basada en el enfoque biográfico, emergen las siguientes categorías: a) Expectativas (proyecto de vida en cinco años). b) Factores explicativos de proyecciones y expectativas. c) Presencia y/o ausencia de proceso de disociación de ideas-creenciasexpectativas (lo que quiero - lo que puedo). d) Sintonía afectiva ligada a idea - creencia expectativa). e) Otra (categoría emergente como resiliencia, estrategias de aprendizaje, habilidades comunicativas, etc.).

El análisis de datos se desarrolló en dos etapas en las que participó un equipo de cinco investigadores. En la primera, se procedió a la lectura colectiva de los relatos escritos de cada participante. Esta lectura no incluyó datos de identificación de participantes ni de liceos de procedencia. A partir de esta lectura, se recopilaron vivencias y proyecciones de vida del estudiantado. En la segunda etapa, se buscó comprender las particularidades de cada relato, mediante una clasificación de las trayectorias en función de los elementos de análisis contenidos en la matriz, los cuales corresponden a las expectativas y las metas a corto, 
mediano y largo plazo elaboradas por quienes participaron, y la vinculación afectiva que presentan las trayectorias, asociadas a las creencias y metas. A partir de este análisis emergen las siguientes categorías (Tabla 2): (1) trayectorias definidas, conceptualizadas como trazados educativos provistos de proyectos de futuro, metas y expectativas claras y definidas, (2) trayectorias difusas, entendidas como trazados educativos oscilantes en torno a proyectos de futuro, metas y expectativas difusas y (3) trayectorias indefinidas, conceptualizadas como trazados educativos con disminución de proyectos de futuro, metas y/o expectativas. La construcción de tipologías de trayectorias denota la complejidad y heterogeneidad en las formas de ser joven, así como también las desventajas estructurales que se inscriben en los diversos recorridos juveniles (Muñiz, Roberti, Deleo y Hasicic, 2013).

En una tercera etapa, se analizaron los relatos escritos en función de las categorías citadas anteriormente con apoyo del programa QDA Miner Lite, software de análisis cualitativo, para la categorización de los relatos según matriz de análisis basada en enfoque biográfico.

A modo de ejemplo, se presentan extractos de relatos de estudiantes participantes del estudio y su categorización a partir de la aplicación de matriz de análisis de contenido (Tabla 2). 
Tabla 2

Matriz de análisis de contenido de Relatos. Chile, región del Bío Bío, año 2015 - 2016

\begin{tabular}{|c|c|c|c|c|c|c|}
\hline $\begin{array}{l}\text { Identificación } \\
\text { de estudiantes } \\
\text { productores de } \\
\text { Relatos }\end{array}$ & $\begin{array}{l}\text { Expectativas - } \\
\text { proyecto de } \\
\text { vida en cinco } \\
\text { años más }\end{array}$ & $\begin{array}{l}\text { Factores } \\
\text { explicativos de } \\
\text { proyecciones y } \\
\text { expectativas }\end{array}$ & $\begin{array}{l}\text { Presencia } \\
\text { y/o ausencia } \\
\text { de proceso } \\
\text { de } \\
\text { disociación } \\
\text { de ideas }\end{array}$ & $\begin{array}{l}\text { Sintonía } \\
\text { afectiva } \\
\text { ligada a idea, } \\
\text { creencia o } \\
\text { expectativa }\end{array}$ & $\begin{array}{l}\text { Otra } \\
\text { (categoría } \\
\text { emergente) }\end{array}$ & $\begin{array}{l}\text { Categorización } \\
\text { Trayectorias: } \\
\text { (1)Definidas } \\
\text { (2) Difusas } \\
\text { (3) Indefinidas }\end{array}$ \\
\hline $\begin{array}{l}\text { Estudiante } \\
\text { mujer } \\
\text { Establecimiento } \\
\text { F - } \\
\text { Extracto Relato }\end{array}$ & $\begin{array}{l}\text { "me encantaría } \\
\text { estudiar } \\
\text { enfermería" }\end{array}$ & $\begin{array}{lrr}\text { “desde } & \text { que } \\
\text { escuché a } & \text { mi } \\
\text { hermana de } & \text { su } \\
\text { carrera y de la } \\
\text { forma en que ella } \\
\text { se relaciona con } \\
\text { sus pacientes } \\
\text {..creo que re he } \\
\text { descubierto } & \text { mi } \\
\text { vocación..." } & \end{array}$ & & $\begin{array}{l}\text { “...poder darle } \\
\text { un buen futuro } \\
\text { a mi pequeña y } \\
\text { que ella se } \\
\text { sienta } \\
\text { orgullosa de la } \\
\text { madre que } \\
\text { tiene..." que }\end{array}$ & $\begin{array}{l}\text { “...en rocial, me } \\
\text { social, me } \\
\text { imagino muy } \\
\text { contenta de } \\
\text { haber } \\
\text { formado } \\
\text { lazos fuertes } \\
\text { con personas } \\
\text { que } \\
\text { comparten } \\
\text { mis } \\
\text { ideales..." }\end{array}$ & $\begin{array}{l}\text { (1) Trayectoria } \\
\text { Definida }\end{array}$ \\
\hline $\begin{array}{l}\text { Estudiante } \\
\text { hombre } \\
\text { Establecimiento } \\
\text { C - } \\
\text { Extracto Relato }\end{array}$ & $\begin{array}{l}\text { "...lo que me } \\
\text { gusta mucho es } \\
\text { la gastronomía } \\
\text {..." }\end{array}$ & 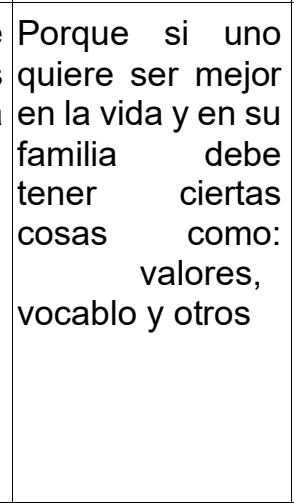 & $\begin{array}{l}\text { “...lo fome es } \\
\text { que si a } \\
\text { futuro tengo } \\
\text { una familia } \\
\text { necesitare un } \\
\text { trabajo que } \\
\text { produzca } \\
\text { dinero y para } \\
\text { ello he } \\
\text { pensado en } \\
\text { la } \\
\text { odontología” }\end{array}$ & $\begin{array}{lr}\text { “...porque } & \text { así } \\
\text { podemos } & \text { ser } \\
\text { mejores } & \\
\text { padres } & \text { y } \\
\text { mejores } & \\
\text { hijos...” } & \end{array}$ & & $\begin{array}{l}\text { (2) Trayectoria } \\
\text { Difusa }\end{array}$ \\
\hline $\begin{array}{l}\text { Estudiante } \\
\text { hombre } \\
\text { Establecimiento } \\
\text { B - } \quad \text { Extracto } \\
\text { Relato }\end{array}$ & \begin{tabular}{|lr} 
“...un & jugador \\
de & futbol \\
profesional & o \\
poder & estudiar \\
en & una \\
universidad”
\end{tabular} & & & $\begin{array}{l}\text { “...enamorarm } \\
\text { e, casarme y } \\
\text { formar una } \\
\text { familia..." }\end{array}$ & \begin{tabular}{l} 
"Poder \\
empezar en \\
un club de \\
Chile luego \\
poder jugar \\
en r los \\
\multicolumn{2}{c}{ mejo } \\
res clubes de \\
Europa... \\
poder llegar \\
a ser el mejor \\
futbolista del \\
mundo..."
\end{tabular} & $\begin{array}{l}\text { (3) Trayectoria } \\
\text { Indefinida }\end{array}$ \\
\hline
\end{tabular}

Fuente: Elaboración propia 


\section{Resultados}

El análisis de las trayectorias estudiantiles se inicia con la identificación de expectativas, elementos explicativos ligados a las expectativas y a la forma en que proyectan sus vidas mediante el establecimiento de metas en el corto, mediano y/o largo plazo.

A partir del análisis de la proyección inicial, el foco de estudio se traslada a la identificación de elementos discursivos disonantes que emerjan a partir del relato de vida escolar. La distancia entre aquello que el estudiante proyecta realizar y expresar en una intención; y lo que la misma persona evalúa poder realizar, se ajusta a la expectativa de acuerdo con la lectura contextual de su dimensión de sujeto sociohistórico. La forma en que cada persona realiza esta lectura contextual da cuenta del habitus o estructuras de pensar, sentir y actuar estudiantil (Wacquant, 2005). La sintonía afectiva ligada a la expectativa del estudiante y la proyección que este realiza fue un aspecto analizado en el relato de vida con base en la función del habitus y su proyección en el sentir y actuar del estudiantado. Mediante la identificación de trayectorias diferenciadas fue posible clasificar las trayectorias vocacionales como definidas, difusas e indefinidas implicadas en la construcción de identidades de las personas entrevistadas.

\subsection{Trayectorias definidas}

Corresponden a las narraciones provistas de experiencias de vida personal, social, laboral y/o académica que el estudiantado realiza respecto de su trayectoria $\mathrm{y}$, en este escenario, se plantean metas en el corto, mediano y largo plazo.

El estudiantado reporta expectativas acerca de lo que esperan lograr en los ámbitos personal, laboral y/o académico. Las proyecciones de vida que construyen se relacionan con el nivel de complejidad y articulación de estas.

...hasta ahora llevo la idea de ser dentista, quizás mis padres no tengan recursos suficientes para pagarme una carrera tan cara, por eso me esfuerzo en mis estudios para lograr obtener una beca y así no tener la necesidad de exigirles tanto a mis padres... (Establecimiento D; mujer).

La elaboración de este relato no solo establece metas, sino que también se adelanta a las dificultades y busca soluciones factibles de realizar, atribuyendo el logro a su esfuerzo personal, en este caso los estudios. 
...me gustaría estar en la Universidad de Concepción estudiando medicina (...) más adelante especializarme en ginecología (...) Me gustaría vivir en el sur con mi pareja, casarme con él y vivir en una casa propia (...) También gustaría tener un buen sueldo para poder ayudar a mi familia económicamente (...) me gustaría tener harto dinero para viajar a otros países, conocer más lugares y así aprender de otras personas... (Establecimiento A; mujer).

Asociado a la elaboración de metas, la aspiración de ingresar a la universidad se encuentra presente como deseo de mejorar su vida. Sin embargo, esta aspiración se concreta de forma imprevista al presentárseles la posibilidad de ingresar a la Educación Superior y apreciar esta opción como una oportunidad y no como una estrategia construida por el propio grupo de jóvenes (López, Mella y Cáceres, 2018).

En los relatos presentados se evidencia una organización en el proyecto de vida de acuerdo a prioridades siguiendo un orden necesario para el alcance de las metas en distintas áreas de su vida. El estudiantado, al trazarse metas y producir proyectos de vida, va incorporando factores explicativos que contribuyen a desarrollar el nivel de motivación adecuado para persistir en la consecución de sus metas. Los elementos a través de los cuales las personas elaboran pautas de significado y apropiación varían de acuerdo con elementos de agencia de las personas considerando su condición sociohistórica y el ejercicio de rol de estudiante de enseñanza media.

......yo creo que tengo actitudes para lograr esta meta que me propuse porque tengo buenas notas y me gusta ayudar a las personas yo creo que tengo vocación para esta área" (Establecimiento L, hombre).

...es una manera de ayudar a las personas y yo ser alguien en la vida, trabajando en lo que me gusta y es mi meta, un logro que quiero y debo alcanzar" (Establecimiento B, mujer).

En su mayoría, los componentes emotivos están relacionados, con el núcleo familiar y el esfuerzo que este ha puesto en el desarrollo de sus jóvenes. Es por esto que, conscientes del esfuerzo implicado, anhelan un futuro que les permita retribuir el esfuerzo de sus padres a través de la recompensa económica, el cuidado, goce y principalmente, la admiración. 
Por otro lado, la vocación asociada al rol profesional es un elemento presente en la elaboración del discurso de los estudiantes con una trayectoria definida. Para este grupo de estudiantes, el reconocimiento de sus capacidades proyectadas al ámbito profesional y al contexto sociocultural de procedencia representa una vía de acceso a sentimientos asociados al logro y realización personal y profesional.

...deseo ejercer esto en las tierras de donde vivo, en un lugar completamente rural, en donde la atención de salud no es muy buena, para poder así ayudar y tratar a las personas que conozco, a mis familiares, ya que así no me alejaría de mis creencias, cultura, para así poder sentirme orgullosa y feliz demostrando lo que he logrado" (Establecimiento A, mujer).

\subsection{Trayectorias difusas}

Comprenden las narraciones provistas de experiencias de vida personal y social que el estudiantado elabora respecto de su proyecto de vida y, en este escenario, se plantean de forma poco clara, metas en el corto, mediano y largo plazo. Este tipo de trayectoria, la trazan en función de la evaluación que realizan respecto de las posibilidades con que cuentan en el mediano y largo plazo y que pueden constituir más de una opción sin que esto implique un proceso de indefinición. El estudiantado presenta expectativas acerca de lo que esperan lograr en los ámbitos personal, laboral o académico. Es decir, se sitúan desde un ámbito para proyectar su vida y, desde ese espacio interno, estructuran su proyección futura en los otros ámbitos de su vida. En este sentido, es relevante para este grupo la experiencia vivencial que posean en el plano personal, laboral o académico, ya que desde este escenario de seguridad y confianza logran elaborar proyectos a futuro.

...lo que me gusta mucho es la gastronomía (...) es que si a futuro tengo una familia necesitare un trabajo que produzca dinero y para ello he pensado en la odontología (Establecimiento C, hombre).

Respecto de las trayectorias difusas del estudiantado y las opciones que estas plantean, las estrategias que han ido construyendo este grupo de jóvenes, a lo largo de la vida, influidas en gran medida por la familia y su historia, se relacionan con el ingreso pronto al mercado de trabajo y no con el ingreso a la universidad. Se habla entonces, de un quiebre de trayectoria en la eventualidad de ingreso del estudiantado a la universidad porque, dentro del camino que recorrerá, los recursos acumulados hasta ese entonces no se corresponden con los que necesita para enfrentar el tránsito universitario (López, Mella y Cáceres, 2018). 
...me proyecto estar estudiando la carrera ojalá la que yo quería (gastronomía, enfermería, obstetricia) o estar en la marina" (Establecimiento O, mujer).

...mi fuerte son las matemáticas y el dibujo y creo que si no es arquitectura es dibujo técnico (...) no quiero ser un vago, solo quiero estudiar para que nunca nada me falte (Establecimiento D, hombre).

Para el estudiantado que se plantea metas de manera difusa, resulta ambivalente la relación que establecen con aquellos elementos motivacionales al momento de dirigir su actuar. En este sentido, tanto las proyecciones elaboradas como los elementos motivacionales asociados oscilan entre sí, teniendo como resultado una proyección con diversos escenarios que, sin embargo, carecen de suficiente consistencia en su elaboración en el tiempo. Esto es concordante con hallazgos que constatan cómo jóvenes de sectores populares estudian menos años y entran a trabajar a edades más tempranas que los de clase alta, manteniéndose la segmentación del sistema de educación superior, pues las universidades más selectivas, lejos de ser más plurales, mantienen estructuras de reproducción de las elites (López, Mella y Cáceres, 2018).

...encuentro que estos tipos de carreras y trabajos [del área de la salud] son muy satisfactorios para mí personalmente, ya que aprendemos de la vida, sabemos conocer nuestro cuerpo y ayudar a mis familiares e hijos y a todos quien necesite mi servicio... (Establecimiento $\mathrm{O}$, hombre).

...las fuerzas armadas de Chile siempre me han llamado la atención desde que era muy niño (Establecimiento $A$, hombre).

...Porque si uno quiere ser mejor en la vida y en su familia debe tener ciertas cosas como: valores, vocablo y otros tipos más de conceptos, porque así podemos ser mejores padres e hijos... (Establecimiento $\mathrm{C}$, hombre).

Los componentes emotivos asociados a las motivaciones son similares a los que se encuentran en la categoría anterior en cuanto al sentimiento de reciprocidad familiar; sin embargo, en estos casos el componente económico emerge como una prioridad. El acto de devolver o retribuir al núcleo familiar, el esfuerzo implicado en el desarrollo personal y social 
de jóvenes, los aproxima a sentimientos de gratitud y retribución que, para este grupo estudiantil, pasa por adquirir una seguridad económica asociada a la proyección de sus vidas en el plano laboral y/o profesional.

...para que mis padres se sientan orgullosos de mí, y en el día de mañana yo devolverles la mano a mis padres y ayudarlos con sus gastos, porque gracias a ellos soy lo que soy (Establecimiento D, hombre).

...mi principal inspiración para lograr ser un profesional y tener un buen sueldo es mi papá quien a pesar de ser un simple obrero ha dado todo por mí y nunca nos ha faltado nada... (Establecimiento $B$, hombre).

...darles la mejor educación a mis hijos ser un ejemplo para ellos (Establecimiento A, hombre).

\subsection{Trayectorias indefinidas}

En el estudiantado que presenta trayectorias vocacionales indefinidas se aprecia una disminución en la elaboración de metas en el corto, mediano y largo plazo. Es importante destacar que este tipo de trayectoria es minoritaria en consideración de la totalidad de los participantes que conforman la muestra.

Este grupo estudiantil se caracteriza por presentar una disminución en la producción de ideas y proyecciones para el mediano y largo plazo. En cuanto a la proyección en el corto plazo, el estudiantado elabora ideas y proyecciones que están principalmente relacionadas con estereotipos sociales y/o mecanismos de evasión. A su vez, estas metas se proyectan sin considerar el proceso necesario para lograrlas.

...un jugador de futbol profesional o poder estudiar en una universidad y poder obtener mi título como profesional luego comenzar a trabajar en alguna empresa y obtener mi propio sueldo (...) enamorarme, casarme y formar una familia, tener hijos, comprarme una casa y tener un auto deportivo también poder viajar por muchas partes del mundo Con mi familia, poder pagarle la educación superior a mis hijos (...) Poder empezar en un club de Chile luego poder jugar en los mejores clubes de Europa seguir creciendo como persona y futbolista para algún día poder dejar a mi país en lo más alto y poder llegar a ser el mejor futbolista del mundo...(Establecimiento B, hombre) 
...No me puedo ver a mí en 5 años más (Establecimiento L, hombre)

...Bueno yo me veo trabajando y además casado y tengo 4 hijos, pero además estoy en la mecánica como profesional pero mi mayor sueño es poder conocer a Demi Lovato y a Daddy Yankee porque soy fans de ellos (Establecimiento $\mathrm{F}$, hombre)

En cuanto a los factores explicativos ligados a las expectativas, estos dan cuenta de una producción poco elaborada en sus relatos debido, en su mayoría, a falta de motivaciones y/o a la dificultad que significa para ellos el visualizar un futuro probable con posibilidades personales, sociales, académicas y/o laborales de las que no tienen conciencia por sus experiencias de vida hasta ahora. Por otro lado, los estereotipos sociales o mecanismos de evasión constituyen para algunos las únicas herramientas que representan movilidad social ante un escenario social y educativo que se les presenta como adverso.

...porque no tengo claro lo que quiero ni lo que puedo hacer... (Establecimiento L, hombre).

...para alentar a las personas, demostrarles que, si se puede, que vean que las cosas pueden ser mejor... (Establecimiento I, mujer).

Los sentimientos asociados a las motivaciones que emergen se relacionan con la soledad y vulnerabilidad que expresan haber vivenciado en la extensión de sus trayectorias. Para este grupo de jóvenes, el escenario adverso es posible de sortear a través del soporte emocional y afectivo que les provee el núcleo familiar, el cual contribuye a amortiguar los sentimientos de soledad. Para ellos, la proyección de metas se vincula afectivamente a la familia que proyectan formar mediante su inserción al mundo del trabajo.

...como hija única quiero ser el orgullo para mi familia en especial para mis padres, que a pesar de mi condición nunca me dejaron sola. En este momento pienso en volar alto y ser alguien en la vida demostrar que soy más fuerte que todos los problemas que se presentan y ser feliz con mis sueños que con esfuerzo y responsabilidad se puede lograr y tenerles una nueva vida y futuro a mis hijos que tendré más adelante y agradecer a mis padres creo y confío en que con mi dedicación lograré muchas cosas y darles lo mejor de mí (Establecimiento G, mujer). 


\section{Conclusiones}

En el presente trabajo se analizaron las trayectorias de vida en función de los elementos estructurales que caracterizan al estudiantado de nivel secundario chileno en situación de vulnerabilidad social. Asimismo se analizó su actuación subjetiva y las expresiones de la capacidad reflexiva llevada a cabo mediante el proceso de toma de decisiones. Los principales hallazgos revelan trayectorias diversificadas según el procesamiento reflexivo en torno al proceso de construcción de proyectos. En efecto, los resultados indican la configuración de trayectorias educativas diversas, la cual se acerca a la conceptualización de trayectorias reales planteada por Terigi, 2009. Al respecto, el estudiantado transita su escolarización de modo complejo, heterogéneo y contingente a sus condicionantes sociohistóricas, lo cual se refleja en la diversidad de trayectos definidos por el estudiantado de secundaria. El grupo de jóvenes, que trazan trayectorias definidas, presentan proyecciones de vida diversificada y compleja respecto a las diferentes opciones que contemplan como posibles. El logro de las metas propuestas es atribuido al esfuerzo personal y al comportamiento de anticipación ante eventuales dificultades que se puedan presentar para su concreción, lo cual resultaría consistente con una racionalidad construida y deconstruida en la definición de identidades juveniles ante condiciones históricas que demandan la reorganización de sus habitus o disposiciones adquiridas. En efecto, el estudiantado que traza estas proyecciones de vida complejas presenta altas y diversas expectativas relacionadas con diversos ámbitos como el personal, escolar y laboral. Este aspecto resulta en concordancia con el lugar preponderante que jóvenes, ubican a los estudios como un componente fundamental en la construcción de sus proyectos de vida.

En el caso de las trayectorias difusas, el estudiantado realiza una evaluación de las posibilidades de proyectar sus vidas en relación con las necesidades afectivas, económicas y sociales actuales, y que esperan satisfacer a partir de la concreción de sus proyectos en el mediano plazo. El componente afectivo de las trayectorias da cuenta del sentimiento de reciprocidad familiar mediado por el componente económico, que emerge como un elemento prioritario. Retribuir al núcleo familiar el esfuerzo implicado en su desarrollo personal y social los aproxima a sentimientos de gratitud y retribución, lo que para este grupo de estudiantes pasa por alcanzar una seguridad económica asociada a la proyección de sus vidas en el plano laboral y/o profesional. Característico de esta generación sería el sentimiento de pragmatismo, ven de manera práctica y vivencial si sus expectativas optimistas tienen correlato con sus experiencias de vida. Se produce, entonces, un desajuste entre esas altas expectativas, las 
que ven difícil de concretar, por los desiguales accesos a las oportunidades sociales, por lo que la forma de resolver la complejidad en sus trayectorias, daría cuenta de reflexiones diversas que colisionan con experiencias de vida ligadas a desventajas estructurales.

El estudiantado que expresa trayectorias indefinidas en la proyección de sus vidas da cuenta de una disminución de proyectos de corto plazo basados en recursos personales para su concreción. Esta constatación daría cuenta de trayectorias reales de tipo no encauzadas o alejadas de las trayectorias teóricas del estudiantado participante, caracterizadas en el mediano y largo plazo por elaborar una visión idealizada de sí mismos, asociada, por ejemplo, a estereotipos sociales mediante la activación de mecanismos de evasión que interfieren en la definición de sus proyectos, en tensión, implicados en este proceso. En el caso de las trayectorias difusas e indefinidas, sería importante profundizar en la función del habitus familiar como elemento comprensivo y articulador de la trayectoria educativa del estudiantado; así como el rol del profesorado en la construcción de proyectos con el estudiantado y cómo se establecen procesos de reflexión docente y lineamientos de mejora que aseguren inclusión, acompañamiento y apoyo emocional que jóvenes de trayectorias indefinidas, requieren para completar ciclo formativo de educación media con proyección de sus vidas en contexto de inclusión social.

Aspectos limitantes por informar lo constituyen el año de inicio del presente estudio que corresponde al 2015, por la dificultad de actualización de datos y la accesibilidad al estudiantado, en aquellos establecimientos educacionales tanto de la provincia de Bío Bío y Concepción, con ubicación geográfica de procedencia rural caracterizada por la fluctuación en la asistencia del estudiantado a las sesiones de intervención del Programa P.A.C.E. Para contrarrestar esta última limitante, los encuentros programados con el estudiantado contemplaron la posibilidad de incorporación de las personas participantes a las distintas etapas de intervención, previa aplicación del consentimiento informado. Dada la naturaleza cualitativa de la investigación podemos señalar que las limitaciones de este estudio se presentan al pretender generalizar los resultados obtenidos. Sin embargo, estos pueden ser profundizados a través de nuevos estudios que indaguen en la construcción de trayectorias juveniles, estudiantes de clase baja y las estrategias que el estudiantado desarrolla para concretar estas aspiraciones y proyectos de vida, tanto de modo individual, familiar y colectivo (grupo social). En este aspecto, tanto la resiliencia como la motivación de cambiar el rumbo de su vida pasan a ser elementos fundamentales que les permitiría asumir la incidencia de sus conductas en las trayectorias de vida. 


\section{Agradecimientos}

Al estudiantado participante del estudio, a la unidad de Dirección de Docencia y a la Universidad de Concepción por el apoyo al programa de Acompañamiento y Acceso Efectivo a la Educación Superior, PACE, iniciativa financiada por el Ministerio de Educación en alianza con la Universidad de Concepción, Chile, de la cual se desprende el presente estudio.

\section{Referencias}

Anders, Jake. (2017). The influence of socioeconomic status on changes in young people's expectations of applying to university. Oxford Review of Education, 43(4), 381-400.Doi: https://doi.org/10.1080/03054985.2017.1329722

Baker, Will., Sammons, Pam., Siraj-Blatchford, Iram., Sylva, Kathy., Melhuish, Edward. y Taggart, Brenda. (2014). Aspirations, education and inequality in England: insights from the Effective Provision of Pre-school, Primary and Secondary Education Project. Oxford Review of Education, 40(5), 525-542. Doi: https://doi.org/10.1080/03054985.2014.953921

Bellei C., Cristian. (2013). El estudio de la segregación socioeconómica y académica de la educación chilena. Estudios pedagógicos (Valdivia), 39(1), 325-345. Doi: http://dx.doi.org/10.4067/S0718-07052013000100019

Bellei, Cristian. (2015). El gran experimento: Mercado y privatización de la educación chilena. Santiago de Chile: LOM ediciones.

Bertaux, Daniel. (1993). De la perspectiva de la historia de vida a la transformación de la práctica sociológica. En José Miguel Marinas y Cristina Santamaría (Eds.), La historia oral: Métodos y experiencias (pp. 19-34). Madrid, España: Debate.

Bertaux, Daniel. (1999). El enfoque metodológico: su validez metodológica, sus potencialidades. Proposiciones, 29. Recuperado de https://www.sitiosur.cl/r.php?id=436

Bourdieu, Pierre. (1984). Distinction. A social critique of the judgement of taste. París: Minuit.

Brunner, José. (2009). Educación superior en Chile: instituciones, mercados y políticas gubernamentales (1967-2007). Chile: Universidad Diego Portales.

Canales, Manuel., Opazo, Antonio. y Camps, Juan Pablo. (2016). Salir de cuarto. Expectativas juveniles en el Chile de hoy. Última década, 24(44), 73-108. Doi: http://dx.doi.org/10.4067/S0718-22362016000100004

Castillo, Jorge. y Cabezas, Gustavo. (2010). Caracterización de jóvenes primera generación en educación superior. Nuevas trayectorias hacia la equidad educativa. Calidad en la Educación, (32), 44-76. Doi http://dx.doi.org/10.31619/caledu.n32.151 
Contreras, Marisol., Corbalán, Francisca. y Redondo, Jesús. (2007). Cuando la suerte está echada: estudio cuantitativo de los factores asociados al rendimiento en la PSU. REICE Revista Iberoamericana sobre Calidad, Eficacia y Cambio en Educación, 5(5), 259-263. Recuperado de https://www.redalyc.org/pdf/551/55121025031.pdf

Cornejo, Marcela. (2006). El enfoque biográfico: Trayectorias, desarrollos teóricos y perspectivas. PSYKHE, 15(1), 95-106. Doi: http://dx.doi.org/10.4067/S0718$\underline{22282006000100008}$

Cornejo, Marcela., Mendoza, Francisca. y Rojas, Rodrigo. (2008). La Investigación con Relatos de Vida: Pistas y Opciones del Diseño Metodológico. PSYKHE, 17(1), 29-39. Doi: http://dx.doi.org/10.4067/S0718-22282008000100004

Dávila, Óscar., Ghiardo, Felipe., y Medrano, Carlos. (2005). Los desheredados: trayectorias de vida y nuevas condiciones juveniles. Valparaíso, Chile: Cidpa.

Dávila, Oscar. y Ghiardo, Felipe. (2005). Juventud, enseñanza secundaria y trayectorias de vida. Educação, 36(1), 67-77.

Doray, Pierre. (2012). De la condition étudiante aux parcours des étudiants: quelques balises théoriques. En France Picardet, Jonas Masdonati, Les parcours d'orientation des jeunes. Dynamiques institutionnelles et identitaires (pp. 51-93). Quebec: Presses de l'Université Laval.

Dubar, Claude. (1998). Trajectoires sociales et formes identitaires. Clarifications conceptuelles et méthodologiques. Sociétés contemporaines, (29), 73-85.

Dubet, François. y Martuccelli, Danilo. (1998). En la escuela. Sociología de la experiencia escolar. Buenos Aires: Editorial Losada.

Espínola, Viola., Claro, Juan Pablo. y Walker, Horacio. (2009). Lineamientos Estratégicos para la Discusión de una Política de Mediano Plazo para la Educación Media. Santiago: Ministerio de Educación/Universidad Diego Portales.

Espinoza, Oscar. (2015). Equidad en el Sistema de Educación Superior en Chile desde la Perspectiva de los Resultados. Propuesta educativa, (43), 46-64. Recuperado de: https://www.redalyc.org/articulo.oa?id=403041714006

García-Huidobro, Juan. (1999). La reforma educacional chilena, 1990-1998. Visión de conjunto. En Juan García-Huidobro (ed.), La reforma educacional chilena (pp. 7-46). Madrid: Editorial Popular.

Gluz, Nora. (2006). La construcción socioeducativa del becario: La productividad simbólica de las políticas sociales en la educación media. Buenos Aires, Argentina: Ed. Instituto Internacional de Planeamiento de la Educación, IIPE-UNESCO.

Gobierno de Chile, Caracterización Socioeconómica Nacional (CASEN). (2015). Encuesta de caracterización socioeconómica nacional. Chile: Ministerio de Desarrollo Social. 
Gobierno de Chile, Junta Nacional de Auxilio Escolar y Becas (JUNAEB). (2017). Indicadores de Vulnerabilidad. Recuperado de https://junaebabierta.junaeb.cl/

Hopenhayn, Martín. y Ottone, Ernesto. (2000). El gran eslabón: educación y desarrollo en el umbral del siglo XXI. Argentina: Fondo de Cultura Económica.

Ley $N^{\circ}$ 19.876. Reforma Constitucional que establece la obligatoriedad y gratuidad de la educación media. Recuperado de https://www.bcn.cl/leychile/navegar?idNorma=210495\&idParte=

López, Irina., Mella, Julio., y Cáceres, Gabriela. (2018). La universidad como ruptura en la trayectoria educativa: experiencias de transición de estudiantes egresados de Enseñanza Media Técnico Profesional que ingresan al Programa Académico de Bachillerato de la Universidad de Chile. Estudios pedagógicos (Valdivia), 44(3), 271-288.

Ministerio de Educación (Mineduc). (2015). Programa de acompañamiento y acceso efectivo a la educación superior (PACE). Santiago de Chile: Ministerio de Educación.

McCulloch, Andrew. (2017). Educational aspirations trajectories in England. British Journal of Educational Studies, 65(1), 69-85. Doi: https://doi.org/10.1080/00071005.2016.1197883

Muñiz, Leticia., Roberti, Eugenia., Deleo, Camila. y Hasicic, Cintia. (2013). Trayectorias laborales en Argentina: una revisión de estudios cualitativos sobre mujeres y jóvenes. Lavboratorio, $\quad$ (25), $\quad 57-79 . \quad$ Recuperado de file:///C:/Users/usuario/AppData/Local/Temp/119-479-1-PB.pdf

Muñoz, Pablo. y Redondo, Amaia. (2013). Inequality and academic achievement in Chile. Cepal Review, (109), 99-114.

Organización para la Cooperación y Desarrollo Económicos (OCDE). (2004). Revisión de Políticas Nacionales de Educación: Chile. París: OCDE.

Rubilar, Gabriela., Muñiz-Terra, Leticia., y Domínguez, Marius. (2019). Sobre el futuro: Narrativas laborales de estudiantes de liceos técnico-profesionales en tres claves de desigualdad. Psicoperspectivas, 18(3), 91-102.

Sepúlveda, Leandro y Valdevenito, María José (2014a), ¿Las cosas claras? Aspiraciones de futuro y proyecto educativo laboral de jóvenes estudiantes secundarios. Estudios Pedagógicos (Valdivia), 40(1), 243-261. Doi: http://dx.doi.org/10.4067/S0718$\underline{07052014000100015}$

Sepúlveda, Leandro y Valdevenito, María José (2014b), Aspiraciones y proyectos de estudiantes de enseñanza técnica-profesional. Polis, 13(38), 597-620. Doi: http://dx.doi.org/10.4067/S0718-65682014000200026

Terigi, Flavia. (2009). Las Trayectorias escolares del problema individual al desafío de política educativo. Buenos Aires: Ministerio de Educación. 
Valenzuela, Juan Pablo., Bellei, Cristián. y De Los Rios, Danae. (2010). Segregación escolar en Chile. En Sergio Martinic y Gregory Elacqua (Eds.), ¿Fin del ciclo? Cambios en la gobernanza del sistema educativo (pp. 209-229). Santiago: UNESCO/Pontificia Universidad Católica.

Wacquant, Loïc. (2005). Habitus. International Encyclopedia of Economic Sociology. London: Routledge.

Zapata, Gonzalo (Coord.), Tejeda, Ivo. y Rojas, Álvaro. (2011). Educación Superior en ChileInforme Nacional. En Centro Interuniversitario de Desarrollo (CINDA), La Educación Superior en Iberoamérica Informe 2011. Educación Superior en Chile 2005-2009. Santiago de Chile, Centro Interuniversitario de Desarrollo (pp. 3-71). Recuperado de https://www.researchgate.net/publication/277584156 Informe de Educacion Superior en Chile 2005-2010 
Revista indizada en

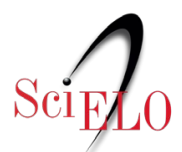
redalyc latindex

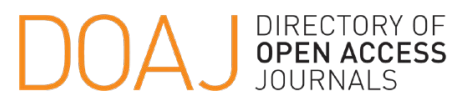

Distribuida en las bases de datos:

- Dialnet
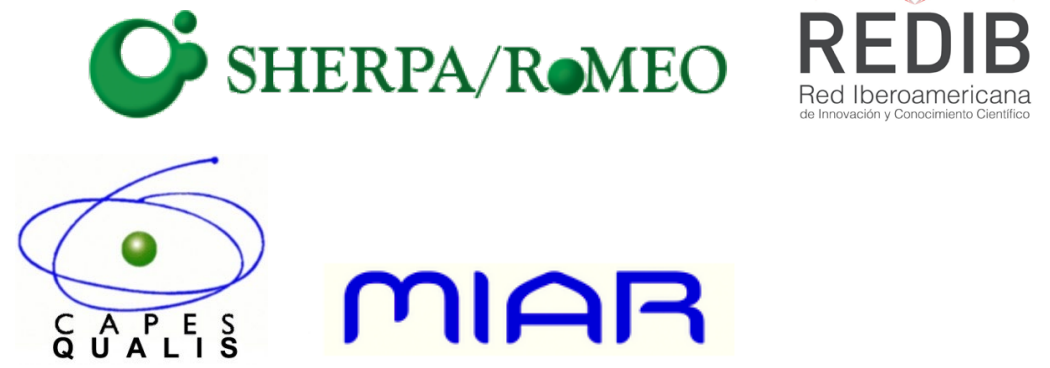

MIAR 